\title{
Excluded-Volume Effects in Dilute Polymer Solutions. V. Butyl Rubber in Various Solvents
}

\author{
Toshiya TsusI \\ Industrial Research Institute of Osaka Prefecture, Osaka, Japan \\ Hiroshi FujIta \\ Department of Polymer Science, Osaka University, Toyonaka, Japan.
}

(Received July 24, 1972)

\begin{abstract}
Seven $\theta$-solvents were found for a sample of commercial butyl rubber which contained $1.40 \mathrm{~mol}-\%$ of isoprene units. Two or three sharp fractions of this rubber sample in a series of solvents, including some of these $\theta$-solvents, were examined by light scattering and viscometry in order to obtain data for the interpenetration function $\Psi$ and the viscosity-radius expansion factor $\alpha_{\eta}$ over a wide range of the statistical-radius expansion factor $\alpha_{\mathrm{s}}$. Plots of $\Psi$ against $\alpha_{\mathrm{s}}{ }^{3}$ were in good agreement with the "best-fit" curve from the authors' previous data and those of Yamakawa, $e t$ al., for other polymer-solvent systems. However, because of scatter, no conclusive information was obtained about the behavior of $\Psi$ in the region of large $\alpha_{\mathrm{s}}{ }^{3}$. Doublelogarithmic plots of $\alpha_{\eta}{ }^{3}$ and $\alpha_{\mathrm{s}}{ }^{3}$, although fitted approximately by a single curve, showed a slight but systematic deviation from the curve which had been proposed previously by Norisuye, et al., on the basis of published data for a number of polymer-solvent systems and later confirmed by Yamakawa, et al., and also by Matsumoto, et al., by measurements on other polymer systems. However, it was clear that the authors' data did not obey the familiar Flory-Fox relation. Finally, the values of $\beta$ (binary cluster integral for unbonded segment-segment interaction) were computed from the observed $\alpha_{\mathrm{s}}$ by making use of the Fujita-Norisuye approximate relation for $\alpha_{\mathrm{S}}$ as a function of the excluded-volume parameter $z$.
\end{abstract}

KEY WORDS Two-Parameter Theory / Excluded-Volume Effect / Interpenetration Function / Expansion Factor / Viscosity-Radius Expansion Factor / Butyl Rubber / Theta-Solvent / Light Scattering / Viscosity /

It is a fundamental requirement of the twoparameter theory of linear homopolymers in dilute solution that the expansion factor $\alpha_{\mathrm{s}}$ and the interpenetration function $\Psi$ depend only on the excluded-volume parameter $z$ and that these functions are universal in the sense that their forms are independent of the chemical nature of the polymer and solvent as well as of the temperature. ${ }^{1}$ Here, in terms of the usual symbols (see previous parts of this series for their significance), the quantities $\alpha_{\mathrm{s}}, \Psi$, and $z$, all being dimensionless, are defined as follows:

and

$$
\begin{gathered}
\alpha_{\mathrm{s}}{ }^{2}=\left\langle S^{2}\right\rangle /\left\langle S^{2}\right\rangle_{0} \\
\Psi=A_{2} M^{2} /\left[4 \pi^{3 / 2} N_{\mathrm{A}}\left\langle S^{2}\right\rangle^{3 / 2}\right]
\end{gathered}
$$

$$
z=\left(4 \pi\left\langle S^{2}\right\rangle_{0}\right)^{-3 / 2} \beta n^{2}
$$

It follows immediately from the above requirement that the values of $\Psi$ for different combinations of polymer, solvent, and temperature should form a single composite curve when plotted against $\alpha_{\mathrm{s}}$.

If the polymer molecule in solution behaves as a sphere impermeable to the flow of solvent, it can be shown, within the framework of twoparameter theory, that the viscosity-radius expansion factor $\alpha_{\eta}$ defined by

$$
\alpha_{\eta}{ }^{3}=[\eta] /[\eta]_{\theta}
$$

should be another universal function of $z$ and hence of $\alpha_{\mathrm{s}}{ }^{1}$

These theoretical predictions were verified, with a fair degree of accuracy, by a number of recent measurements on well-defined samples of 


\section{T. Tsuji and H. Fujita}

linear homopolymers, but the observed values of $\Psi$ and $\alpha_{\eta}$ as functions of $\alpha_{\mathrm{s}}$ agreed only approximately with the expressions provided by the existing two-parameter theories. The discrepancy is primarily attributable to the approximate nature of these theories, but it is apparent that more accurate and extensive experimental data are needed in order to reach unambiguous conclusions concerning the excluded-volume effects in dilute polymer solutions.

In the studies so far reported, the parameter $z$, and hence $\alpha_{\mathrm{s}}$, was varied mainly by changing the molecular weight of the polymer sample and/or the temperature of the system. A similar variation of $z$, however, could be achieved by changing the kind of solvent, because the parameter $\beta$ involved in the expression for $z$ depends on the solvent power of a given solvent. $^{1}$ In fact, this idea was employed, though not extensively, by previous investigators, who used for a given polymer at least two kinds of solvent which differed in solvent power. However, when plotted against the observed $\alpha_{\mathrm{s}}{ }^{3}$, the experimental values of $\Psi$ and $\alpha_{\eta}{ }^{3}$ obtained in different solvents, especially in highly nonideal ones, did not always form a continuous curve as satisfactorily as one might expect. This result, which may be chiefly attributable to the experimental difficulty of measuring $\left\langle S^{2}\right\rangle$ and $A_{2}$ with precision, keeps one from transforming the observed data to the definitive conclusion about the behavior of $\Psi\left(\alpha_{\mathrm{s}}\right)$ and $\alpha_{\eta}\left(\alpha_{\mathrm{s}}\right)$ in good solvents, where the values of $\alpha_{\mathrm{s}}{ }^{3}$ are large.

Because of such a situation, it seemed important to reinvestigate more specifically the effects of solvent power on these functions, and the experimental work reported in the present paper was initiated. Here a commercial butyl rubber was chosen as the polymer sample, and $\Psi, \alpha_{\mathrm{s}}$, and $\alpha_{\eta}$ were determined for a variety of solvent conditions so chosen that a wide range of $z$ might be covered. It is hoped that the experimental results reported here will be complementary to the previous measurements in which $z$ was varied mainly by changing the molecular weight of the sample.

Butyl rubber is a copolymer of isobutylene and isoprene, so one might consider that it is not very relevant for testing the two-parameter theory of polymer solutions. However, as will be seen below, since the content of isoprene units in the rubber sample used was only 1.40mol\%, it was not unreasonable to postulate that the copolymer effects, if any, were essentially negligible and that, as far as most of the dilute solution properties were concerned, the polymer used would behave as if it were a homopolymer of isobutylene. In the subsequent description, the polymer is simply referred to as butyl rubber, with its isoprene content not specified unless needed.

\section{EXPERIMENTAL}

\section{Polymer Fractions}

Butyl 217, a commercial product kindly furnished by Esso Chemicals, was chosen as the starting material for the present work. Its isoprene content, estimated by the method of Gallo, et al., ${ }^{2}$ was found to be 1.40 -mole $\%$ when two independent determinations were averaged. Recent NMR measurements by $\mathrm{Yu}$ and Field $^{3}$ have shown that the isoprene units incorporated in butyl rubber chains were predominantly in the $1-4$ configuration. It was assumed that this was also the case with the sample used in this report, although this postulate has nothing to do directly with the discussion developed below.

First, the given product was precipitated from a benzene solution by addition of acetone, and the polymer recovered by freeze-drying from a cyclohexane solution of the precipitate. The viscosity-average molecular weight, $\bar{M}_{v}$, of the sample so purified was $4.2 \times 10^{5}$ when estimated from the intrinsic viscosity, $[\eta]$, in benzene at $22.8^{\circ} \mathrm{C}$ (a $\theta$-solvent for butyl rubber, as will be seen below) by using the relationship $[\eta]=11.5 \times$ $10^{-4} M^{0.5}$ (see below).

The purified sample was divided into seven fractions, designated here as $\mathrm{C}-1, \mathrm{C}-2, \ldots, \mathrm{C}$ 7 , by the same theta elution column method as employed in the authors' previous work on polyisobutylene. ${ }^{4}$ Benzene was used as the eluting solvent. Six fractions but C-1 were then subjected to successive fractional precipitation in order to extract their main fraction. Phase separation was produced either by adding iso- 
propanol to or by lowering the temperature of a benzene solution. Five members were selected from a number of subfractions obtained, and were coded CM-2, CM-3, ., CM-6 in order of increasing $\bar{M}_{v}$. After freeze-drying from cyclohexane solutions containing appropriate amounts of an antioxidant $2,2^{\prime}$-methylene bis(4-methyl-6-tert butyl-phenol) they were stored at about $-20^{\circ} \mathrm{C}$ until needed for use.

\section{Solvents}

Ideal Solvents. Benzene at $24.5^{\circ} \mathrm{C}$ is the bestknown ideal (or $\theta$ ) solvent for polyisobutylene, ${ }^{4}$ but it is not suitable for light-scattering study of this polymer since, as has been noted previously, the refractive index increment of the system is negligibly small. ${ }^{4}$ Therefore, in their recent work on polyisobutylene, Matsumoto, et $a l .,{ }^{4}$ sought another $\theta$-solvent which is free from this disadvantage and found that isoamyl isovalerate (IAIV) at $22.1^{\circ} \mathrm{C}$ was adequate. The authors anticipated that both benzene and IAIV at room temperature could also be $\theta$-solvents for butyl rubber, and began the present work by determining the exact $\theta$-temperatures of these solvents.

For this purpose, osmotic second virial coefficients of suitably chosen fractions in benzene and in IAIV were measured at three temperatures near $23^{\circ} \mathrm{C}$. Figures 1 and 2 show the osmotic pressure data obtained. From these one finds by interpolation that benzene at 22.8 $( \pm 0.3)^{\circ} \mathrm{C}$ and IAIV at $21.0( \pm 1.0)^{\circ} \mathrm{C}$ are $\theta$ solvents for butyl rubber. The $\theta$-temperature in benzene is about $2^{\circ} \mathrm{C}$ lower than those reported for polyisobutylene. This might well be due to the fact that benzene acts as a good solvent for the isoprene units in butyl rubber.

To discover more $\theta$-solvents for butyl rubber the solubility of the polymer in about 40 different kinds of organic liquid was examined. From the results several were that appeared promising for the purpose of this report and the intrinsic viscosities of one or two fractions of them measured at several suitably chosen temperatures. When the values obtained were compared with those of the same fractions in the $\theta$-solvent (benzene at $22.8^{\circ} \mathrm{C}$ ), it seemed quite probable that the first three of the five solvents listed below would have $\theta$-temperatures

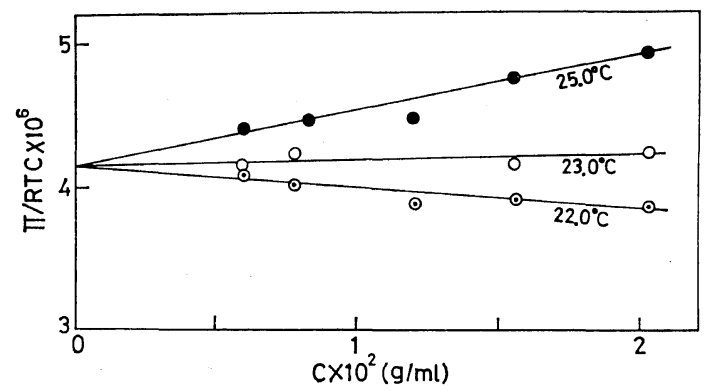

Figure 1. Reduced osmotic pressure $\pi / R T c$ plotted against polymer concentration $c$ for butyl rubber fraction $\mathrm{B}-5$ in benzene at $22.0,23.0$, and $25.0^{\circ} \mathrm{C}$.

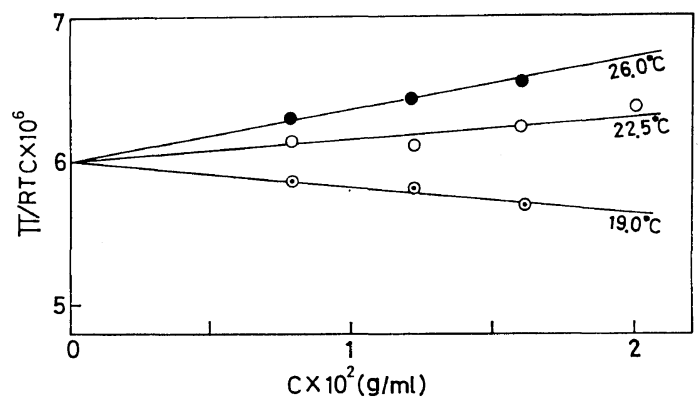

Figure 2. Reduced osmotic pressure $\pi / R T c$ plotted against polymer concentration $c$ for butyl rubber fraction CM-2 in isoamyl isovalerate at 19.0, 22.5, and $26.0^{\circ} \mathrm{C}$.

Table I. $\theta$-solvents and associated $\theta$-temperatures for butyl rubber (containing 1.40-mol\% isoprene units)

\begin{tabular}{lc}
\hline \multicolumn{1}{c}{$\theta$-solvent } & $\theta$-temperature, ${ }^{\circ} \mathrm{C}$ \\
\hline Isoamyl isovalerate & 21.0 \\
$n$-Amyl $n$-butyrate & 22.0 \\
Benzene & 22.8 \\
Isoamyl benzyl ether & 23.7 \\
Isoamyl $n$-butyrate & 28.0 \\
$n$-Butyl $n$-butyrate & 46.2 \\
3-Methyl 5-heptanone & 55.5 \\
\hline
\end{tabular}

in the vicinity of $25^{\circ} \mathrm{C}$, while the last two would have such temperatures near $50^{\circ} \mathrm{C}$ :

isoamyl benzyl ether (IABE), $n$-amyl $n$-butyrate (NANB), isoamyl $n$-butyrate (IANB), 3methyl 5-heptanone (MH), n-butyl $n$-butyrate (NBNB).

To determine the exact $\theta$-temperatures, therefore, osmotic-pressure measurements were made in each of these five solvents at three tempera- 
tures encompassing the $\theta$-temperature expected for each. By either interpolation or extrapolation of the osmotic second virial coefficients obtained, the $\theta$-temperatures were determined as shown in Table I, wherein those for benzene and IAIV are also included.

It is probably worth noting that, according to the solubility tests, $n$-butyl acetate, $n$-amyl acetate, isoamyl acetate, and mesityl oxide were all precipitants for butyl rubber, despite the fact that they had been reported to be solvents for polyisobutylene.

Nonideal Solvents. It was essential for this study to obtain a series of solvents whose $\beta$ for butyl rubber varies over a wide range and spaced at suitable intervals. Further, it was necessary that those solvents should have specific refractive index increments, $\mathrm{d} n / \mathrm{d} c$, large enough for light-scattering measurements to be made with accuracy. As is well known there is no means of evaluating $\beta$ directly. The quantity $\alpha_{\eta}$ was therefore substituted as a measure of its strength, and, by trial and error processes, solvents were sought which might give (for the chosen polymer fractions) a series of $\alpha_{\eta}$ values that are different and at suitable spacings. For the estimate of $\mathrm{d} n / \mathrm{d} c$ in such solvents the Gladstone-Dale equation was applied, substituting the refractive index of polyisobutylene for that of butyl rubber. After a number of trials, the following five were finally chosen as nonideal solvents appropriate for the physical measurements:

isoamyl $n$-caprylate (IANC), isoamyl ether (IAE), $n$-hexane, methylcyclohexane, cyclohexane

The values of $\alpha_{\eta}$ and $\mathrm{d} n / \mathrm{d} c$ for fraction CM-6 in these solvents are indicated in Table II. Cyclohexene was found to be intermediate in $\alpha_{\eta}$ between $n$-hexane and methylcyclohexane, but it was discarded because of its extremely bad smell. Investigations to find solvents poorer than the above five, however, were unsuccessful. Therefore, one was forced to obtain data corresponding to such solvent conditions by the use of $\theta$-solvents which were shifted away from their $\theta$-temperatures. IANB and IAIV were used for this purpose. Table II also contains the relevant data for these solvents.
Table II. $\alpha_{\eta}^{3}$ and $\mathrm{d} n / \mathrm{d} c$ of butyl rubber in various solvents

\begin{tabular}{lcll}
\hline \multicolumn{1}{c}{ Solvent } & Temp, ${ }^{\circ} \mathrm{C}$ & $\alpha_{\eta}{ }^{\mathrm{a}}$ & $\mathrm{d} n / \mathrm{d} c^{\mathrm{b}}$ \\
\hline Isoamyl $n$-butyrate & $28.0(\theta)$ & 1.0 & $0.117_{0}$ \\
& 40.0 & 1.14 & $0.120_{0}$ \\
Isoamyl isovalerate & 19.95 & & $0.109_{7}$ \\
& 32.2 & & $0.112_{6}$ \\
& 40.0 & 1.25 & \\
& 43.4 & & $0.115_{5}$ \\
& 51.8 & & $0.118_{8}$ \\
& 58.9 & 1.38 & \\
Cyclohexane & 25.0 & 3.49 & $0.101_{6}$ \\
Methylcyclohexane & 25.0 & 3.20 & $0.106_{0}$ \\
$n$-Hexane & 25.0 & 2.08 & $0.167_{6}$ \\
Isoamyl ether & 25.0 & 1.92 & $0.119_{6}$ \\
Isoamyl $n$-caprylate & 25.0 & 1.57 & 0.0972 \\
\hline
\end{tabular}

a Values for fraction CM-6.

b Values for light of the wavelength $436 \mathrm{~m} \mu$.

Each of these solvents was thoroughly purified by a standard procedure, and aliphatic esters were put to use within 5 days and others within 7 days after distillation. These periods were determined by preliminary experiments in which the change in refractive indices of the solvents was measured as a function of time.

\section{Solutions}

The polymer was dissolved in a given solvent at room temperature, with continuous stirring of the mixture, except in the cases of IAIV and IANB for which the temperature of dissolution was raised to $40^{\circ} \mathrm{C}$.

For viscosity measurements a series of four concentrations were prepared by successive dilution of the most concentrated solution. In the light-scattering experiments the antioxidant was first removed from the fractions by precipitating the polymer from a benzene solution with the addition of acetone. With each of the fractions so purified, two solutions of different concentrations were independently made up, and five concentrations in total prepared by diluting them directly in the light-scattering cell, except in the case of IANB solutions for which the desired concentrations were obtained from a single original solution. Except for cyclohexane solutions, the concentrations were chosen so that the ratio of $90^{\circ}$ intensities of scattered light for solution and solvent fell in the range 
10-20. Before introduction into the light-scattering cell, both solutions and solvents were carefully cleaned by centrifugation at $20000 \mathrm{~g} .{ }^{4}$

\section{Light-Scattering Measurement}

Determination of $\mathrm{d} n / \mathrm{d} c$. A differential refractometer of the modified Schulz-Cantow type was employed. ${ }^{6}$ The instrument constant was determined with aqueous solutions of highly purified $\mathrm{KCl}$, with the data of $\mathrm{Kruis}^{7}$ being chosen as the reference. The data were taken with unpolarized light of $435.8 \mathrm{~m} \mu$. The values found, in units of milliliters per gram, were as follows: $0.117_{0}$ and $0.120_{0}$ in IANB at $28.0^{\circ}$ and $40.0^{\circ} \mathrm{C}$, respectively; $0.109_{7}, 0.112_{6}, 0.115_{5}$, and $0.118_{8}$ in IAIV at $19.95^{\circ}, 32.2^{\circ}, 43.4^{\circ}$, and $51.8^{\circ} \mathrm{C}$, respectively; $0.101_{6}$ in cyclohexane at $25.0^{\circ} \mathrm{C} ; 0.106_{0}$ in methylcyclohexane at $25.0^{\circ} \mathrm{C}$; $0.167_{6}$ in $n$-hexane at $25.0^{\circ} \mathrm{C} ; 0.119_{6}$ in IAE at $25.0^{\circ} \mathrm{C} ; 0.0972$ in IANC at $25.0^{\circ} \mathrm{C}$.

Light-Scattering Photometry. The same apparatus and experimental procedures as in the previous study on polyisobutylene were employed. ${ }^{4}$ Except in IANB and IAIV, the data were taken at $25.0^{\circ} \mathrm{C}$ only. In IANB at $28.0^{\circ} \mathrm{C}$, all five fractions of the CM-series were examined, but, in other solvents, only two or three of them were subjected to the measurement. Raw data were analyzed in terms of Berry's modification $^{8}$ of the Zimm $\operatorname{method}^{9}$ to evaluate $\bar{M}_{w}$, $\left\langle S^{2}\right\rangle$, and $A_{2}$.

\section{Osmotic-Pressure Measurement}

A Mechrolab 502 High Speed Membrane osmometer fitted with an adequately conditioned gel-cellophane was used. Measurements were made, on one hand, to determine the exact $\theta$ temperatures of the solvents listed in Table I from the temperature dependence of $A_{2}$ and, on the other hand, to evaluate the $\bar{M}_{n}$ of the fractions CM-2, CM-3, and CM-6.

\section{Viscosity Measurement}

A capillary viscometer of the Fitzsimons type $^{10}$ was used. It had an upper bulb of about $2 \mathrm{~m} l$ and a flow time of $170.4 \mathrm{sec}$ for benzene at $22.8^{\circ} \mathrm{C}$. Correction for kinetic energy was not necessary because of the design of the viscometer. Shear-rate effects, if any, could be ignored as far as the relatively small specific viscosities encountered in the present measurements were concerned. The extrapola- tion to infinite dilution was made in the same way as in the previous studies of this series.

\section{RESULTS}

\section{Light Scattering}

Osmotic pressure measurements indicated the $\theta$-temperature to be $27.5^{\circ} \mathrm{C}$ for the butyl rubber in IANB. In order to acertain this result an attempt was first made to determine the lightscattering $A_{2}$ for the fraction CM-5 in this solvent at $26^{\circ}, 28^{\circ}$, and $30^{\circ} \mathrm{C}$, and values $-0.06 \times 10^{-4}, 0$, and $0.06 \times 10^{-4}$ were obtainedall in units of $\mathrm{ml}-\mathrm{mol} / \mathrm{g}^{2}$, at these temperatures. Though the difference from $27.5^{\circ} \mathrm{C}$ seemed to be within experimental error, it was decided to take $28.0^{\circ} \mathrm{C}$ as the correct $\theta$-temperature for the system butyl rubber-IANB. Additional support for this decision is presented in Figure 3, wherein one can see that light-scattering plots for all five fractions of the CM-series in IANB at $28.0^{\circ} \mathrm{C}$ and at zero scattering angle are horizontal, giving zero for $A_{2}$.

Figure 4 illustrates, with the data for fraction

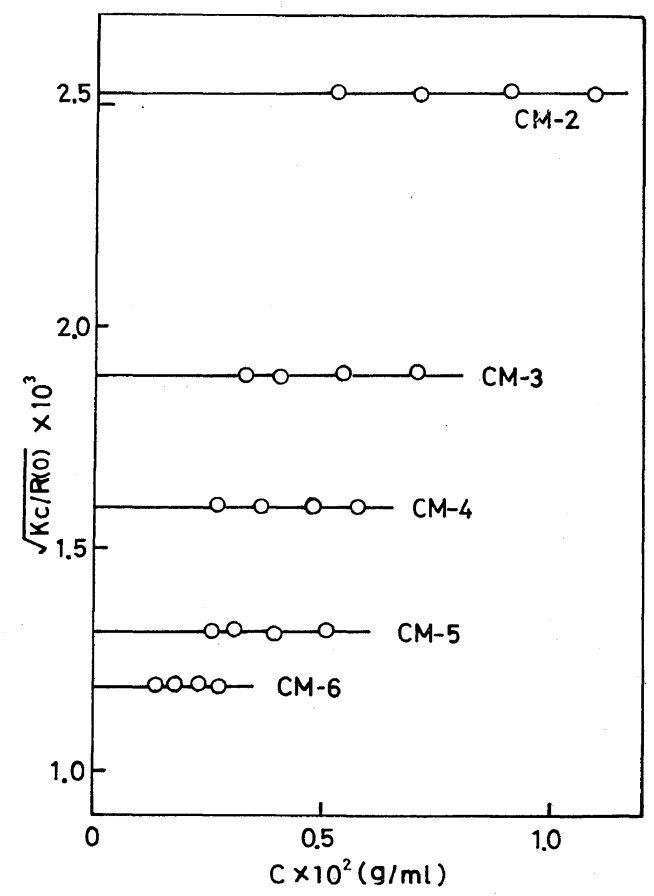

Figure 3. Plots of $[K c / R(0)]^{1 / 2}$ vs. polymer concentration $c$ for butyl rubber fractions in isoamyl $n$-butyrate at $28.0^{\circ} \mathrm{C}$. 


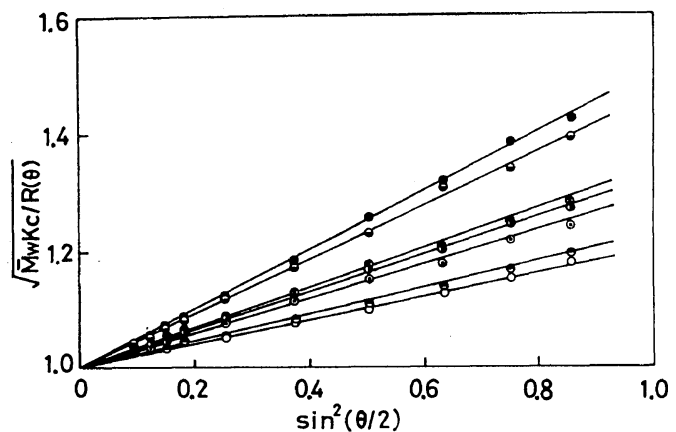

Figure 4. Plots of $\left[\bar{M}_{w} K c / R(\theta)\right]^{1 / 2} v s . \sin ^{2}(\theta / 2)$ for fraction CM-6 in various solvents: $O$, cyclohexane $\left(25^{\circ} \mathrm{C}\right) ; \ominus$, methylcyclohexane $\left(25^{\circ} \mathrm{C}\right) ;(\otimes), n$-hexane $\left(25^{\circ} \mathrm{C}\right)$; $\bigcirc$, isoamyl ether $\left(25^{\circ} \mathrm{C}\right)$; $\odot$, isoamyl $n$ caprylate $\left(25^{\circ} \mathrm{C}\right)$; $\Theta$, isoamyl isovalerate $\left(58.9^{\circ} \mathrm{C}\right)$; $\mathrm{O}$, isoamyl $n$-butyrate $\left(28^{\circ} \mathrm{C}\right)$.

CM-6, how the square root of the reciprocal reduced intensity of scattered light at zero polymer concentration varied with scattering angle in the seven solvents examined. The slopes of the indicated straight lines give values of $\left\langle S^{2}\right\rangle$ for this fraction in these solvents at the temperatures indicated. Concentration dependence of the corresponding quantity at zero scattering angle for the same systems is displayed in Figure 5. The values of $A_{2}$ for these systems can be computed from the slopes of the lines indicated.

Numerical data from light-scattering and osmotic-pressure measurements are collected in Table III. The following points may be noted.

(1) Though available only for fractions CM2, CM-3, and CM-6, the ratio of $\bar{M}_{w}$ to $\bar{M}_{n}$ is quite close to unity, suggesting that these fractions were quite homogeneous with respect to molecular weight. One would expect that this was also the case with two other fractions CM-4 and CM-5.

(2) For each of the fractions CM-4, CM-5, and CM-6, the values of $\bar{M}_{w}$ obtained in different solvents (except for CM-6 in IANC at $25^{\circ} \mathrm{C}$ ) stand in good agreement, as should be the case theoretically. In the ensuing discussion, the $\bar{M}_{w}$ values determined in IANB at $28.0^{\circ} \mathrm{C}$ will be used as the molecular weights of the fractions examined.

(3) The observed values of $\left\langle S^{2}\right\rangle^{1 / 2}$ in IANB at $28.0^{\circ} \mathrm{C}$, denoted hereafter by $\left\langle S^{2}\right\rangle_{0}{ }^{1 / 2}$, are

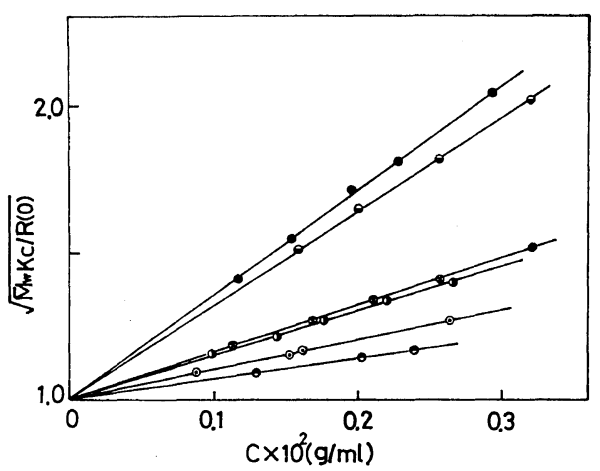

Figure 5. Plots of $\left[\bar{M}_{w} K c / R(0)\right]^{1 / 2} v s$. polymer concentration $c$ for fraction CM-6 in various solvents. The symbols are the same as in Figure 4.

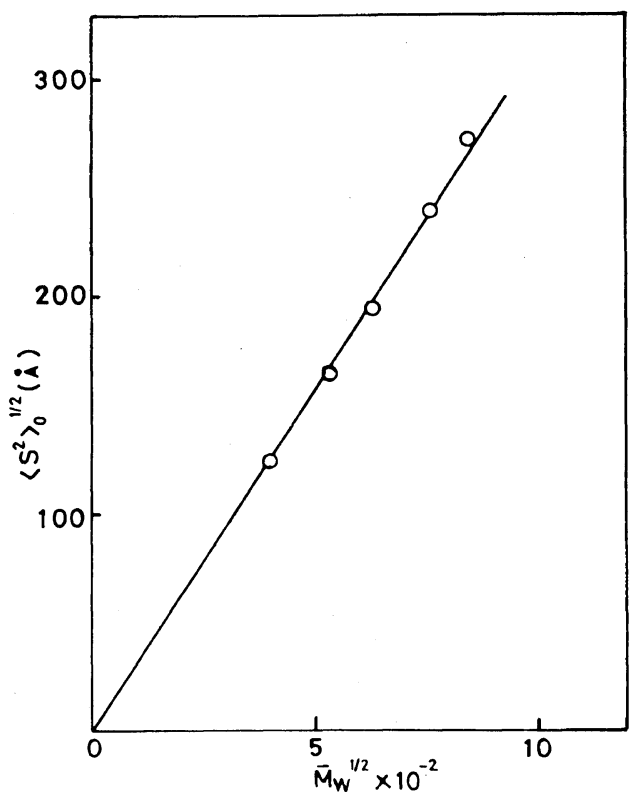

Figure 6. Plots of $\left\langle S^{2}\right\rangle_{0}^{1 / 2}$ vs. $\bar{M}_{w^{1 / 2}}$ for butyl rubber fractions in isoamyl $n$-butyrate at $28.0^{\circ} \mathrm{C}$.

plotted against $\bar{M}_{w}{ }^{1 / 2}$ in Figure 6 . The fact that the data points are fitted by a straight line passing through the origin is consistent with the finding that IANB at $28.0^{\circ} \mathrm{C}$ is a $\theta$-solvent for the butyl rubber. The straight line indicated is reprensented by

$$
\left\langle\boldsymbol{S}^{2}\right\rangle_{0}=9.8_{6} \times 10^{-18} \bar{M}_{w}
$$

where $\left\langle S^{2}\right\rangle_{0}$ is expressed in square centimeters. This equation may be compared to the relation $\left\langle S^{2}\right\rangle_{0}=9.5_{2} \times 10^{-18} \bar{M}_{w}$ reported by Matsumoto, 
Butyl Rubber

Table III. Light-scattering and osmotic-pressure data for butyl rubber

\begin{tabular}{|c|c|c|c|c|c|c|}
\hline Solvent ${ }^{\mathrm{a}}$ & Temp, ${ }^{\circ} \mathrm{C}$ & Fraction & $\bar{M}_{w} \times 10^{-4}$ & $\bar{M}_{n} \times 10^{-4}$ & $\left\langle S^{2}\right\rangle^{1 / 2}, \AA$ & $A_{2} \times 10^{4}, \mathrm{ml} \mathrm{mol} / \mathrm{g}^{2}$ \\
\hline \multirow[t]{5}{*}{ IANB } & 28.0 & CM-6 & 71.1 & $65.8^{b}$ & $27_{1}$ & 0 \\
\hline & & CM-5 & 57.6 & & $23_{8}$ & 0 \\
\hline & & CM-4 & 39.5 & & $19_{3}$ & 0 \\
\hline & & CM-3 & 28.1 & $28.2^{\mathrm{c}}$ & $16_{3}$ & 0 \\
\hline & & CM-2 & 15.7 & $16.6^{\mathrm{d}}$ & $12_{3}$ & 0 \\
\hline IANB & 40.0 & CM-6 & 71.0 & & 290 & $0.41_{2}$ \\
\hline \multirow[t]{2}{*}{$\mathrm{CH}$} & 25.0 & CM-6 & 70.7 & & $42_{8}$ & 4.99 \\
\hline & & CM-5 & 55.9 & & $36_{6}$ & $5.2_{4}$ \\
\hline \multirow[t]{3}{*}{$\mathrm{MCH}$} & 25.0 & CM-6 & 71.5 & & $41_{0}$ & 4.49 \\
\hline & & CM-5 & 56.3 & & $35_{1}$ & $4.7_{7}$ \\
\hline & & CM-4 & 38.4 & & $27_{5}$ & $5.3_{2}$ \\
\hline \multirow[t]{2}{*}{ NH } & 25.0 & CM-6 & 71.9 & & $35_{2}$ & $2.2_{6}$ \\
\hline & & CM-5 & 57.9 & & $30_{5}$ & $2.4_{7}$ \\
\hline \multirow[t]{2}{*}{ IAE } & 25.0 & CM-6 & 71.9 & & $33_{9}$ & $2.0_{7}$ \\
\hline & & CM-5 & 57.0 & & $30_{4}$ & $2.3_{3}$ \\
\hline \multirow[t]{2}{*}{ IANC } & 25.0 & CM-6 & 67.1 & & $32_{2}$ & $1.5_{1}$ \\
\hline & & CM-5 & 57.1 & & $28_{2}$ & $1.6_{7}$ \\
\hline IAIV & 58.9 & CM-6 & 71.9 & & $30_{3}$ & 0.96 \\
\hline IAIV & 40.0 & CM-6 & 72.5 & & $29_{5}$ & 0.58 \\
\hline
\end{tabular}

a Abbreviations: IANB, isoamyl $n$-butyrate; $\mathrm{CH}$, cyclohexane; $\mathrm{MCH}$, methylcyclohexane; $\mathrm{NH}, n$ b In benzene at $25^{\circ} \mathrm{C}$. hexane; IAE, isoamyl ether; IANC, isoamyl $n$-caprylate; IAIV, isoamyl isovalerate.

c In benzene at $22.8^{\circ} \mathrm{C}$.

d In IAIV at $21.0^{\circ} \mathrm{C}$.

et al.,$^{4}$ for polyisobutylene in a $\theta$-solvent, IAIV at $22.1^{\circ} \mathrm{C}$. The numerical coefficient in eq 5 yields a value of $1.8_{7}$ for the conformational parameter $\sigma .^{11}$

\section{Intrinsic Viscosity}

Table IV summarizes numerical results from the viscosity measurements. Conventional log$\log$ plots of $[\eta] v s . \bar{M}_{w}$ in four $\theta$-solvents are collected in Figure 7. It is seen that the set of data points for each solvent is fitted by a straight line whose slope is 0.5 , and the following Houwink-Mark-Sakurada relations can be derived.

For benzene at $22.8^{\circ} \mathrm{C}$ :

$$
[\eta]=1.1_{5} \times 10^{-3} \bar{M}_{w}{ }^{1 / 2}
$$

For isoamyl benzyl ether at $23.7^{\circ} \mathrm{C}$ :

$$
[\eta]=1.0_{8} \times 10^{-3} \bar{M}_{w}{ }^{1 / 2}
$$

For 3-methyl 5-heptanone at $55.5^{\circ} \mathrm{C}$ :

$$
[\eta]=1.0_{9} \times 10^{-3} \bar{M}_{w}{ }^{1 / 2}
$$

For isoamyl $n$-butyrate at $28.0^{\circ} \mathrm{C}$ :

$$
[\eta]=1.1_{4} \times 10^{-3} \bar{M}_{w}{ }^{1 / 2}
$$

Here $[\eta]$ is expressed in deciliters per gram.

Close agreement of the numerical coefficients in these relations lends support to the generally accepted notion that the intrinsic viscosity of a linear (homo) polymer under $\theta$-conditions is essentially independent of the kind of solvent and temperature. However, it is debatable whether the small difference observed in these coefficients may simply be attributed to the uncertainty which arises in fitting a straight line to the plotted points. In order to check this point, it is advantageous to make a direct comparison of the observed [ $\eta]$ values for a particular fraction in solvents which are different chemically but have approximately the same $\theta$-temperatures and also those in solvents which are similar chemically but have different $\theta$-temperatures. IABE and benzene were selected as examples of the former kind of solvents. Table IV indicates that the $[\eta]$ values for fraction CM-3 in these solvents at the respective $\theta$-temperatures are 0.568 (for IABE) and 0.614 
T. Tsuji and H. Fujita

Table IV. Viscosity data for butyl rubber in various solvents (I)

\begin{tabular}{|c|c|c|c|c|c|}
\hline Solvent & Temp, ${ }^{\circ} \mathrm{C}$ & Fraction & $\bar{M}_{w} \times 10^{-5^{\mathrm{a}}}$ & {$[\eta], \mathrm{d} l / \mathrm{g}$} & $k^{\prime}$ \\
\hline \multirow[t]{6}{*}{ Isoamyl $n$-butyrate } & $28.0(\theta)$ & $\mathrm{C}-2$ & $1.5_{7}$ & 0.446 & 0.50 \\
\hline & $28.0(\theta)$ & C-3 & $2.8_{1}$ & 0.613 & 0.51 \\
\hline & $28.0(\theta)$ & C-4 & $3.9_{5}$ & 0.730 & 0.53 \\
\hline & $28.0(\theta)$ & C-5 & $5.7_{6}$ & 0.863 & 0.56 \\
\hline & $28.0(\theta)$ & C-6 & $7.1_{1}$ & 0.942 & 0.59 \\
\hline & 40.0 & C-6 & $7.1_{1}$ & 1.07 & 0.53 \\
\hline \multirow[t]{4}{*}{ Isoamyl isovalerate } & $21.0(\theta)$ & $\mathrm{C}-3$ & $2.8_{1}$ & 0.625 & 0.57 \\
\hline & $21.0(\theta)$ & $\mathrm{C}-4$ & $3.9_{5}$ & 0.738 & 0.59 \\
\hline & 40.0 & $\mathrm{C}-6$ & $7.1_{1}$ & 1.17 & 0.52 \\
\hline & 58.9 & C-6 & $7.1_{1}$ & 1.30 & 0.52 \\
\hline \multirow[t]{2}{*}{$n$-Butyl $n$-butyrate } & $46.2(\theta)$ & C-3 & $2.8_{1}$ & 0.600 & 0.57 \\
\hline & $46.2(\theta)$ & C-4 & $3.9_{5}$ & 0.720 & 0.55 \\
\hline$n$-Amyl $n$-butyrate & $22.0(\theta)$ & $\mathrm{C}-3$ & $2.8_{1}$ & 0.605 & 0.57 \\
\hline \multirow[t]{10}{*}{ Benzene } & $22.8(\theta)$ & $\mathrm{C}-2$ & $1.5_{7}$ & 0.470 & 0.55 \\
\hline & $22.8(\theta)$ & $\mathrm{C}-3$ & $2.8_{1}$ & 0.614 & 0.61 \\
\hline & $22.8(\theta)$ & $\mathrm{C}-4$ & $3.9_{5}$ & 0.729 & 0.60 \\
\hline & $22.8(\theta)$ & C-5 & $5.7_{6}$ & 0.847 & 0.62 \\
\hline & $22.8(\theta)$ & C-6 & $7.1_{1}$ & 0.964 & 0.56 \\
\hline & 25.0 & $\mathrm{C}-2$ & $1.5_{7}$ & 0.490 & 0.52 \\
\hline & 25.0 & C-3 & $2.8_{1}$ & 0.653 & 0.58 \\
\hline & 25.0 & $\mathrm{C}-4$ & $3.9_{5}$ & 0.775 & 0.57 \\
\hline & 25.0 & C-5 & $5.7_{6}$ & 0.919 & 0.58 \\
\hline & 25.0 & C-6 & $7.1_{1}$ & 1.04 & 0.55 \\
\hline
\end{tabular}

a Values obtained from light-scattering measurements in isoamyl $n$-butyrate at $28.0^{\circ} \mathrm{C}$.

Viscosity data for butyl rubber in various solvents (II)

\begin{tabular}{cccccc}
\hline Solvent & Temp, ${ }^{\circ} \mathrm{C}$ & Fraction & $\bar{M}_{w} \times 10^{-5^{\mathrm{a}}}$ & {$[\eta], \mathrm{d} l / \mathrm{g}$} & $\mathrm{k}^{\prime}$ \\
\hline Isoamyl benzyl ether & $23.7(\theta)$ & $\mathrm{C}-2$ & $1.5_{7}$ & 0.440 & 0.56 \\
& $23.7(\theta)$ & $\mathrm{C}-3$ & $2.8_{1}$ & 0.568 & 0.54 \\
& $23.7(\theta)$ & $\mathrm{C}-4$ & $3.9_{5}$ & 0.685 & 0.55 \\
& $23.7(\theta)$ & $\mathrm{C}-5$ & $5.7_{6}$ & 0.829 & 0.57 \\
3-Methyl 5-heptanone & $23.7(\theta)$ & $\mathrm{C}-6$ & $7.1_{1}$ & 0.895 & 0.71 \\
& $55.5(\theta)$ & $\mathrm{C}-2$ & $1.5_{7}$ & 0.437 & 0.56 \\
& $55.5(\theta)$ & $\mathrm{C}-3$ & $2.8_{1}$ & 0.591 & 0.67 \\
& $55.5(\theta)$ & $\mathrm{C}-4$ & $3.9_{5}$ & 0.676 & 0.70 \\
Cyclohexane & $55.5(\theta)$ & $\mathrm{C}-5$ & $5.7_{6}$ & 0.816 & 0.67 \\
& $55.5(\theta)$ & $\mathrm{C}-6$ & $7.1_{1}$ & 0.930 & 0.66 \\
Methylcyclohexane & 25.0 & $\mathrm{C}-5$ & $5.7_{6}$ & 2.74 & 0.38 \\
& 25.0 & $\mathrm{C}-6$ & $7.1_{1}$ & 3.29 & 0.35 \\
n-Hexane & 25.0 & $\mathrm{C}-4$ & 3.95 & 1.94 & 0.35 \\
Isoamyl ether & 25.0 & $\mathrm{C}-5$ & $5.7_{6}$ & 2.57 & 0.33 \\
Isoamyl $n$-caprylate & 25.0 & $\mathrm{C}-6$ & $7.1_{1}$ & 3.02 & 0.33 \\
& 25.0 & $\mathrm{C}-5$ & $5.7_{6}$ & 1.70 & 0.36 \\
& 25.0 & $\mathrm{C}-6$ & $7.1_{1}$ & 1.96 & 0.36 \\
\hline
\end{tabular}

a Values obtained from light-scattering measurements in isoamyl $n$-butyrate at $28.0^{\circ} \mathrm{C}$. 


\section{Butyl Rubber}

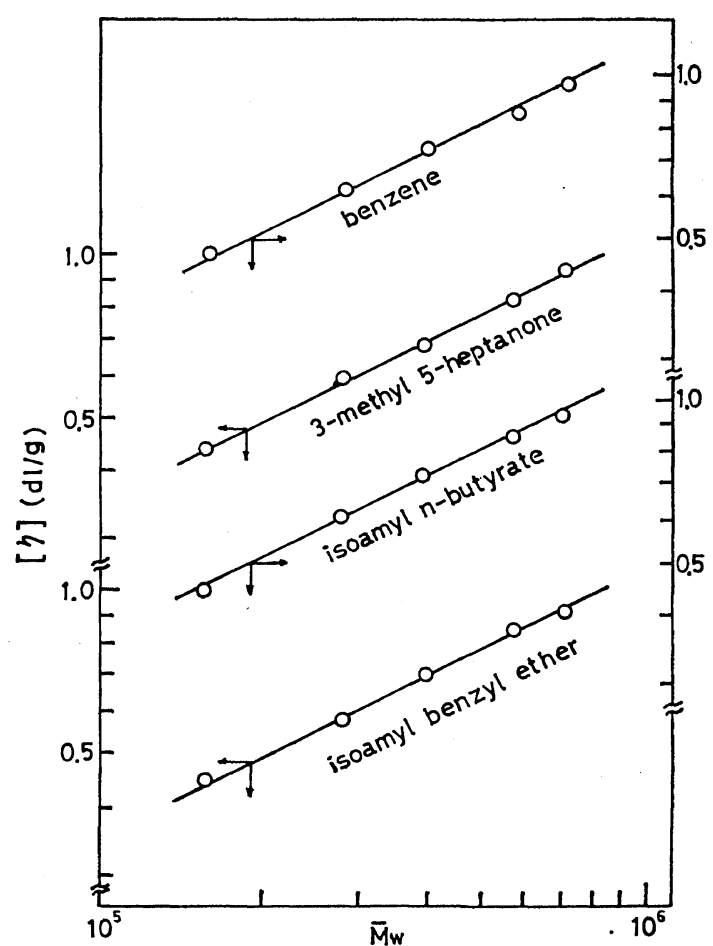

Figure 7. Double logarithmic plots of $[\eta] v s . \bar{M}_{w}$ for butyl rubber fractions: in benzene at $22.8^{\circ} \mathrm{C}$, in 3-methyl 5 -heptanone at $55.5^{\circ} \mathrm{C}$, in isoamyl $n$ butyrate at $28.0^{\circ} \mathrm{C}$, and in isoamyl benzyl ether at $23.7^{\circ} \mathrm{C}$.

(for benzene). The difference between the two values certainly exceeds the limits of experimental error, and it may well be attributed to the chemical difference between the two solvents. Next, four aliphatic esters IAIV, NANB, IANB, and NBNB were chosen as examples of the latter kind of solvent. Their $\theta$-temperatures are different by $25^{\circ} \mathrm{C}$ at the largest, and the $[\eta]$ values of fraction CM-3 in them at the respective $\theta$ temperatures vary from 0.600 to 0.625 . Roughly, these $[\eta]$ values decrease as $\theta$-temperature becomes higher. Because of the chemical similarity of the four esters, one might ascribe this trend to the temperature dependence of the unperturbed dimensions of the polymer chain. However, this idea seems to be irrelevant, because the temperature coefficient of the unperturbed dimensions estimated from these data is too large in comparison with that known for polyisobutylene from thermoelastic measurements. ${ }^{12}$ Thus, it appears that the variation of $[\eta]$ among the four esters, though almost comparable to the accuracy of measurement, may also be attributed to the difference in their molecular structures.

\section{Relation between $\Psi$ and $\alpha_{\mathrm{s}}{ }^{3}$}

Values of $\Psi$ for all combinations of molecular weight, solvent species, and temperature treated in the present experiment are plotted against the corresponding $\alpha_{\mathrm{s}}{ }^{3}$ in Figure 8 . Here, for $\left\langle S^{2}\right\rangle_{0}$ required in the computation of $\alpha_{\mathrm{s}}$, the experimental values obtained in IANB at $28.0^{\circ} \mathrm{C}$ have been used. For clarity, the data in different solvents are distinguished by different symbols.

Unfortunately, the data points do not form as smooth a single curve as one might wish to obtain from the theoretical prediction mentioned in the Introduction. This result, however, should not be taken as evidence pointing to the breakdown of the two-parameter theory. Rather it is to be considered in the light of the fact that values of $\Psi$ are very sensitive to the errors in measurements of $\bar{M}_{w},\left\langle S^{2}\right\rangle$, and $A_{2}$. Especially, in the region where the change in $\Psi$ with $\alpha_{\mathrm{s}}{ }^{3}$ is gradual, even slight inaccuracies of these experimental quantities give rise to an absurd scatter of the points. If one takes this situation into account, these results may rather be regarded as verifying, if not definitely, the theoretical requirement of the two-parameter theory that $\Psi$ depends only on $\alpha_{\mathrm{s}}$. The solid line in the figure is a tentative fit to the plotted points, whereas the dashed curve represents the best fit, proposed by Norisuye, et al., to their own data on polychloroprene ${ }^{13}$ and Berry's data

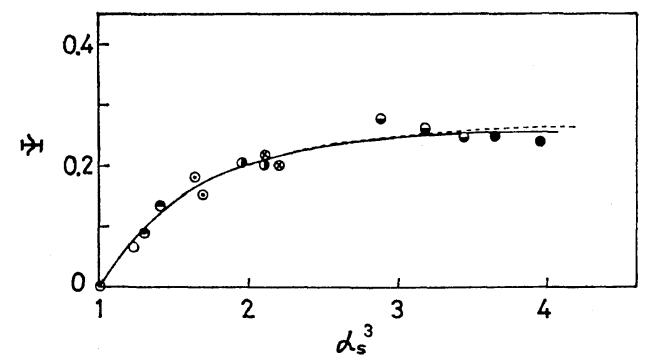

Figure 8. Plots of $\Psi$ vs. $\alpha_{\mathrm{s}}{ }^{3}$ for butyl rubber fractions. The symbols are the same as in Figure 4. Solid line, a best fit to the present data. Dashed line, a best fit to data of Norisuye, et al., for polychloroprene and of Berry for polystyrene. 


\section{T. Tsuji and H. Fujita}

on polystyrene. ${ }^{8}$ Confirmation of the latter curve has been provided by recent measurements on several other polymer-solvent systems. ${ }^{1}$ The close agreement of the solid line with it may be taken as further support for its general validity. However, it should be noted that the present study still failed to determine with complete certainty the seemingly asymptotic behavior of $\Psi$ at large $\alpha_{s}$, which, as has been pointed out in the Introduction, is one of the as-yet unsolved problems in the field of polymer solution study.

Relation between $A_{2} \bar{M}_{w} /[\eta]$ and $\alpha_{\mathrm{s}}$

The dimensionless quantity $A_{2} \bar{M}_{w} /[\eta]$ is known to behave similarly as the interpenetration function. Its values calculated from the present data are plotted against $\alpha_{\mathrm{s}}{ }^{2}-1$ in Figure 9. It is seen that the data points are fitted quite well by a single curve (solid line) which appears to level off at a constant value of $110 \pm 5$. This feature may be taken to mean that viscosityradius expansion factor $\alpha_{\eta}$ depends only on $\alpha_{\mathrm{s}}$ or that the polymer coil behaves as if it were impermeable to solvent. For comparison, the best fit curves to the data for polychloroprene ${ }^{14}$ and to those for polyisobutylene ${ }^{4}$ are shown, respectively, by chain-dotted and dashed lines in Figure 9. The present data appear to follow more closely the chain-dotted line, but it is probably not very meaningful to speak of the

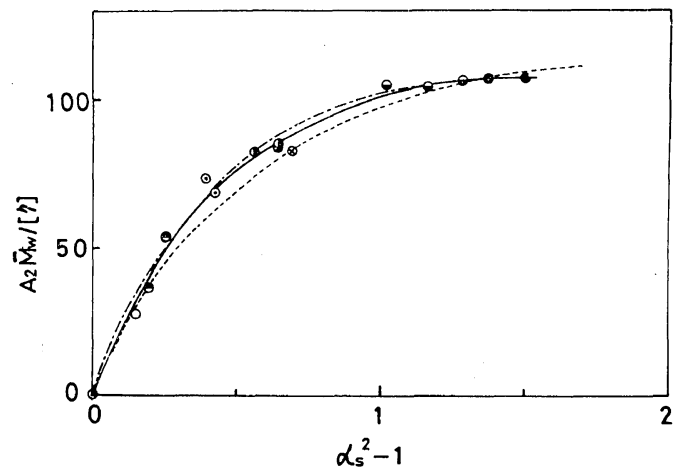

Figure 9. Plots of $A_{2} \bar{M}_{w} /[\eta]$ vs. $\alpha_{\mathrm{s}}{ }^{2}-1$ for butyl rubber fractions. The symbols are the same as in Figure 4. Solid line, a best fit to the present data. Chain-dotted line, a best fit to data by Kawahara, et al., for polychloroprene. Dashed line, a best fit to data by Matsumoto, et al., for polyisobutylene. small disparity in the three curves if one considers the experimental errors involved in the measurements of $\bar{M}_{w}$ and $A_{2}$, especially the latter. In passing, Yamakawa and his collaborators, ${ }^{15,16}$ working with other polymers, obtained data which conformed excellently to the chain-dotted line.

Relation between $\alpha_{\eta}{ }^{3}$ and $\alpha_{\mathrm{s}}{ }^{3}$

As mentioned above, the data shown in Figure 9 suggest that $\alpha_{\eta}$ for the polymer-solvent systems investigated here depend only on $\alpha_{\mathrm{s}}$. In order to check this suggestion explicitly, the values of $\alpha_{\eta}{ }^{3}$ computed for all experimental conditions are plotted double-logarithmically against the corresponding values of $\alpha_{\mathrm{s}}{ }^{3}$ in Figure 10. The data points do not follow a single curve as satisfactorily as one might expect, but one may fit them approximately by a solid line as indicated. This line is slightly above the chain-dotted curve which represents the best fit to the data of Kawahara, et al. ${ }^{14}$ for polychloroprene and those of Takashima, et al. ${ }^{17}$ for $\operatorname{poly}\left(p\right.$-bromostyrene). Norisuye, et al. ${ }^{18}$ showed that this chain-dotted line also runs through the center of a relatively narrow band

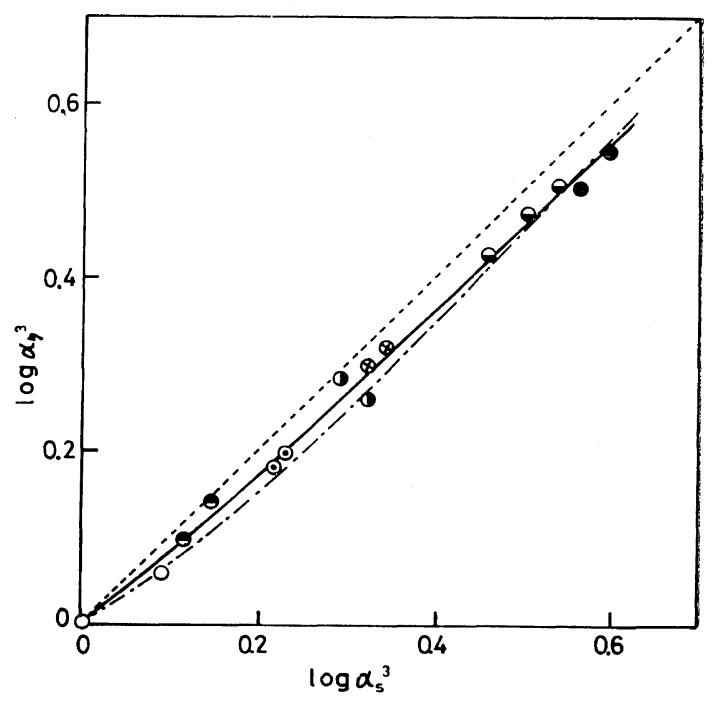

Figure 10. Double logarithmic plots of $\alpha_{\eta}{ }^{3} v s$. $\alpha_{\mathrm{s}}{ }^{3}$ for butyl rubber fractions. The symbols are the same as in Figure 4. Solid line, a best fit to the present data. Chain-dotted line, a best fit to data by Kawahara, et al., for polychloroprene. Dotted line, the Flory-Fox relation $\alpha_{\eta}{ }^{3}=\alpha_{\mathrm{s}}{ }^{3}$. 


\section{Butyl Rubber}

in which a great number of data points for several other polymer-solvent systems appear. More recent measurements by Matsumoto, et $a l .,{ }^{4}$ on polyisobutylene and those by Yamamoto, et al.,$^{16}$ on polystyrene also provided data which lent support to the empirical fit of Norisuye, et al. $^{13}$ Probably, the slight, but somewhat systematic deviation of the present data from the chain-dotted line should not be taken too seriously, but may be attributed for the moment to the lack of precision in the authors' measurements of $\left\langle S^{2}\right\rangle$, especially at values of $\alpha_{\mathrm{s}}$ close to unity.

The dashed line in Figure 10 represents the familiar Flory-Fox relation $\alpha_{\eta}{ }^{3}=\alpha_{\mathrm{s}}{ }^{3}$. It is obvious that these data do not obey it.

\section{The Parameter $\beta$}

Finally, the parameter $\beta$ was computed from the present data. For this purpose, first the excluded-volume parameter $z$ was calculated from observed $\alpha_{\mathrm{s}}$ by making use of the approximate relation derived by Fujita and Norisuye $^{19}$ (note that this is essentially identical to the Yamakawa-Tanaka ${ }^{20}$ equation formulated by use of a different mathematical approach) and then the results substituted into eq 3 , together with the directly measured values for $\left\langle S^{2}\right\rangle_{0}$ and $n$ ( $=\bar{M}_{w} / m$, with $m$ being the molecular weight of the isobutylene residue).

Table V. Values of $\beta$ calculated from the Fujita-Norisuye theory for $\alpha_{\mathrm{s}}(z)$

\begin{tabular}{lcll}
\hline \multicolumn{1}{c}{ Solvent } & $\begin{array}{c}\text { Temp, } \\
{ }^{\circ} \mathrm{C}\end{array}$ & Fraction & $\begin{array}{c}\beta \times 10^{23} \\
\mathrm{~cm}^{3}\end{array}$ \\
\hline Cyclohexane & 25.0 & CM-6 & 1.9 \\
Methylcyclohexane & 25.0 & CM-5 & 1.8 \\
& 25.0 & CM-6 & 1.4 \\
& 25.0 & CM-5 & 1.3 \\
n-Hexane & 25.0 & CM-4 & 1.3 \\
& 25.0 & CM-6 & 0.51 \\
Isoamyl ether & 25.0 & CM-5 & 0.51 \\
& 25.0 & CM-6 & 0.37 \\
Isoamyl n-caprylate & 25.0 & CM-5 & 0.52 \\
& 25.0 & CM-6 & 0.27 \\
Isoamyl isovalerate & 25.0 & CM-5 & 0.26 \\
& 58.9 & CM-6 & 0.13 \\
Isoamyl $n$-butyrate & 40.0 & CM-6 & 0.10 \\
& 40.0 & CM-6 & 0.067 \\
\hline
\end{tabular}

a Value reported by Matsumoto, et al., for polyisobutylene. ${ }^{4}$
The values of $\beta$ so obtained for various nonideal systems investigated here are shown in fourth column of Table $V$. It is seen that, as required from the definition of $\beta$, these values for fixed solvent conditions are essentially independent of the molecular weight of the polymer, except in one solvent, isoamyl ether. However, one should not take this result as implying that the listed values are correct in absolute magnitude, because the Fujita-Norisuye relation ${ }^{19}$ used is only approximate. If the modified Flory equation $^{21}$ were used for the calculation of $z$, different values of $\beta$ would be obtained.

In Table $\mathrm{V}$ is quoted, for comparison, the value of $\beta$ derived for polyisobutylene in cyclohexane at $25^{\circ} \mathrm{C}$ by Matsumoto, ${ }^{4}$ who used a method of evaluation similar to that described above. Its small difference from this report's value for butyl rubber may be ascribed to the errors committed by the two groups of authors in the measurements of $\left\langle S^{2}\right\rangle$.

Acknowledgments. One of the authors (T.T.) wishes to thank the Head of the Industrial Research Institute of Osaka Prefecture for the permission to leave his duty for the completion of this work. His thanks are also due to Dr. $K$. Kawahara and other colleagues at the Department of Polymer Science, Osaka University, for their assistance and advice given in many aspects of the present experiment.

\section{REFERENCES}

1. H. Yamakawa, "Modern Theory of Polymer Solutions," Harper \& Row, New York, N.Y., 1971.

2. S. G. Gallo, H. K. Wiese, and J. F. Nelson, Ind. Eng. Chem., 40, 1277 (1948).

3. $\mathrm{H}$. $\mathrm{Yu}$ and J.E. Field, Rubber Chem. and Technol., 42, 392 (1969).

4. T. Matsumoto, N. Nishioka, and H. Fujita, $J$. Polym. Sci. Part A-2, 10, 23 (1972).

5. Polymer Handbook, J. Brandrup and E. H. Immergut, Ed., Interscience Publishers, New York, N.Y., 1966, Chapter IV, p 187.

6. K. Okita, A. Teramoto, K. Kawahara, and H. Fujita, J. Phys. Chem., 72, 278 (1968).

7. A. Kruis, Z. Phys. Chem., 34Z, 13 (1936).

8. G. C. Berry, J. Chem. Phys., 44, 4550 (1966).

9. B. Zimm, ibid., 16, 1099 (1948).

10. O. Fitzsimons, Ind. Eng. Chem., Anal. Ed., 7, 


\section{T. Tsujr and H. Fujita}

345 (1935).

11. M. Kurata and W. H. Stockmayer, Advan. Polym. Sci., 3, 196 (1963).

12. A. Ciferri, C. A. Hoeve, and P. J. Flory, $J$. Amer. Chem. Soc., 83, 1015 (1961).

13. T. Norisuye, K. Kawahara, A. Teramoto, and H. Fujita, J. Chem. Phys., 49, 4330 (1968).

14. K. Kawahara, T. Norisuye, and H. Fujita, ibid., 49, 4339 (1968).

15. G. Tanaka, S. Imai, and H. Yamakawa, ibid., 52, 2639 (1970).
16. A. Yamamoto, M. Fujii, G. Tanaka, and $\mathbf{H}$. Yamakawa, Polymer J., 2, 799 (1971).

17. K. Takashima, G. Tanaka, and H. Yamakawa, ibid. 2, 245 (1971).

18. T. Norisuye, K. Kawahara, and H. Fujita, Polym. Lett., 6, 849 (1968).

19. H. Fujita and T. Norisuye, J. Chem. Phys., 52, 1115 (1970).

20. H. Yamakawa and G. Tanaka, ibid., 47, 3991 (1967).

21. W. H. Stockmayer, Makromol. Chem., 35, 54 (1960). 